\title{
Thelytoky and sex determination in the Hymenoptera:
}

\section{mutual constraints}

Christoph Vorburger

Institute of Integrative Biology, ETH Zürich, Universitätstrasse 16, 8092 Zürich, Switzerland and

EAWAG, Swiss Federal Institute of Aquatic Science and Technology, Überlandstrasse 133;

8600 Dübendorf, Switzerland

running title: thelytoky and sex determination

correspondence: Christoph Vorburger

Institute of Integrative Biology, ETH Zürich \& EAWAG

Überlandstrasse 133

8600 Dübendorf

Switzerland

phone: +41587655196

fax: +41 587655315

e-mail: christoph.vorburger@eawag.ch

This document is the accepted manuscript version of the following article:

vorburger, C. (2014). Thelytoky and sex determination in the hymenoptera: mutual constraints. Sexual Development, 8(1), 50-58. http://doi.org/10.1159/000356508 


\section{Abstract}

2

3 The Hymenoptera show a high propensity for transitions from arrhenotokous reproduction

4 (diploid females develop from fertilised eggs, haploid males from unfertilized eggs) to

5 thelytokous reproduction (diploid females develop from unfertilised eggs). However, the

6 evolution of thelytoky is frequently constrained by the sex determination system. Under the

7 ancestral system, complementary sex determiation (CSD), the constraint results from the

8 production of diploid males by thelytokous females. The magnitude of this constraint

9 depends on the cytological mechanism of thelytoky, determining the rate at which

10 thelytokous lines lose heterozygosity, and on whether a single locus or multiple loci are

11 involved in CSD. In this review I discuss how diploid male production in the case of CSD or

12 other constraints in the case of alternative sex determination systems may impede transitions

13 to thelytoky, but I also show that under particular (and presumably rare) circumstances, the

14 production of diploid males will promote rather than hamper the evolution of thelytoky. I

15 further argue that the constraints between the evolution of thelytoky and sex determination

16 may be mutual, because once thelytoky has evolved, it can impact on the sex determination.

17 Finally, I encourage researchers to exploit the frequent occurrence of thelytoky as an

18 opportunity to learn more about the mechanisms of sex determination in the Hymenoptera.

20 Keywords: automixis, complementary sex determination, diploid males, Hymenoptera,

21 thelytoky 
Introduction

Insects are the most diverse group of animals on our planet. Unsurprisingly for such a

26 huge group, insects comprise a mind-boggling variety of genetic systems [Normark, 2003].

27 As one of the largest orders within the insects, the Hymenoptera are remarkably

28 homogeneous in this respect. All show haplodiploidy, which in turn may explain a number of important phenomena in this order, such as the evolution of eusociality, sex ratio

manipulation or the high tolerance to inbreeding [Hamilton, 1964; Werren, 1993; Shuker and

31 West, 2004]. Haplodiploidy is linked to the ability to bring unfertilized eggs to development

32 and to a sex determination system that does not rely on sex chromosomes [Kraaijeveld,

33 2009]. The former makes hymenopterans prone to transitions from arrhenotoky to thelytoky

34 and the latter may - depending on the cytological mechanism involved in thelytoky - lead to

35 interesting clashes with their sex determination [Engelstädter, 2008]. Here I discuss how the

36 sex determination system can constrain the evolution of thelytoky in the Hymenoptera via

37 different mechanisms that are mostly well understood, and I offer more speculative

38 arguments as to how thelytoky can influence the evolution of the sex determination system.

39 That is, I propose that the evolution of thelytoky and the evolution of sex determination may

40 be linked by mutual constraints in the Hymenoptera.

41

\section{Haplodiploidy as a pre-adaptation to thelytoky}

43

The potential for parthenogenetic reproduction is widespread in insects. It is latent even in many groups that are diplodiploid and generally reproduce sexually, such as locusts, cockroaches or mayflies [Pardo et al., 1995; Ball, 2001; Corley et al., 2001]. The spontaneous production of some diploid eggs that can develop into females without fertilization is referred 
to as tychoparthenogenesis. It can be considered as a strategy that ensures some reproductive success even when females are mate-limited [Gerritsen, 1980]. Tychoparthenogenesis has also been proposed to be a starting point that may lead to the evolution of obligate

51 parthenogenesis through a positive feedback loop in that increasing rates of

52 tychoparthenogenesis skew the sex ratio towards females, which will further increase mate

53 limitation and thus selection for parthenogenetic reproduction [Schwander et al., 2010].

54 However, such transitions are often hampered by reduced fitness returns from parthenogenetic reproduction [e.g. Corley and Moore, 1999]. This is typically due to developmental constraints [Corley et al., 1999; Sekine and Tojo, 2010], which can be caused

57 by deviations from the maternal ploidy level, by inbreeding depression resulting from 58 increased homozygosity and by other effects [reviewed by Engelstädter, 2008]. In short, many insects are capable of parthenogenesis, but most are not very good at it. This is different in the Hymenoptera (and presumably other haplodiploids): Males develop from unfertilized eggs (arrhenotoky); they are routinely produced by parthenogenesis and this has been so for at least 200 million years [Grimaldi and Engel, 2005]. Clearly, hymenopterans are good at it. As a consequence, hymenopterans are particularly prone to transitions from arrhenotoky to thelytoky, the production of diploid daughters from unfertilized eggs. Such transitions may be induced by Wolbachia and other bacterial endosymbionts that selfishly manipulate their host's reproduction [Duron et al., 2008; Werren et al., 2008] or by thelytoky-

67 inducing alleles in the insect's own genome [Lattorff et al., 2005; Sandrock and Vorburger,

68 2011]. Whatever the actor, it can usurp a pre-existing cellular machinery that allows successful development from unfertilized eggs.

A second reason why transitions to thelytoky occur readily in hymenopterans is the strong

71 selection against recessive deleterious mutations. Such mutations cannot accumulate under

72 haplodiploidy - at least if they are not female-limited - because they are not protected by a 
73 wild-type allele in the haploid males. Males thus act as a filter against recessive deleterious

74 alleles, making hymenopterans more tolerant of inbreeding than diplodiploid insects [Werren,

75 1993]. This is important because thelytoky in hymenopterans is frequently achieved via some

76 form of automixis, which equates to uniparental inbreeding. Many species can even tolerate

77 its most extreme form, gamete duplication (Fig. 1), which leads to $100 \%$ homozygosity in the

78 offspring [Stouthamer and Kazmer, 1994]. However, thelytoky with automixis can be

79 detrimental if the sex determination system is such that some or all of the asexually produced

80 offspring develop into diploid males, which frequently have low fitness in hymenopterans

81 [Cook and Crozier, 1995; Holloway et al., 1999; Zayed and Packer, 2005]. This can also be

82 interpreted as a - potentially severe - form of inbreeding depression and is explained in more

83 detail below. Note that similar constraints may also apply to diplodiploid insects, particularly

84 those with female heterogamety (ZW sex chromosomes), in which gamete duplication would

85 lead to either ZZ (male) or WW (inviable) offspring [Cook and Butcher, 1999].

87 Sex determination in the Hymenoptera

88

Based on his studies of sex determination in the parasitoid wasp Bracon hebetor, Whiting

[Whiting, 1933, 1939, 1943] proposed a model of complementary sex determination (CSD)

governed by a single locus (sl-CSD). Under this model, zygotes develop into females if they carry two different alleles at a proposed sex determination locus, but into males if they carry one allele (hemizygotes) or two copies of the same allele (homozygotes). This model can be extended to two or multiple loci (ml-CSD), under which heterozygosity at one or more loci leads to female development, whereas individuals that are hemizygous or homozygous at all sex determination loci develop into haploid or diploid males, respectively [Snell, 1935;

97 Crozier, 1971]. Both models predict the production of diploid males under inbreeding, albeit 
at different rates. In a sl-CSD system, already the first generation of sib-matings should lead to the occurrence of diploid males in half of the broods. In a ml-CSD system, several generations of inbreeding may be required to observe diploid male production, depending on the number of loci involved [Cook, 1993; de Boer et al., 2008].

The occurrence of CSD has been confirmed by experimental inbreeding for many species 103 [van Wilgenburg et al., 2006; Heimpel and de Boer, 2008] and has become a hallmark of hymenopteran reproduction. Phylogenetic analyses do indeed support that CSD is the ancestral mechanism of sex determination in the Hymenoptera, but they do not allow any firm conclusions as to whether sl-CSD or ml-CSD was the ancestral state [Asplen et al., 107 2009]. In the honey bee, which exhibits sl-CSD, the genetic regulation of sex determination 108 has been largely elucidated [Beye et al., 2003; Hasselmann et al., 2008; Gempe et al., 2009]. 109 It turned out that the $c s d$ gene arose by gene duplication from the feminizer (fem) gene, which is an ortholog of transformer (tra), the principal sex determining gene in flies.

111 Heterozygosity at $c s d$ is required to produce the active proteins that induce female-specific 112 splicing of the fem mRNAs. The Fem protein then directs female-specific splicing of the 113 doublesex (dsx) gene and thus female development [Gempe and Beye, 2011].

114 However, CSD is not universal in the Hymenoptera. Many species and presumably some entire clades such as the superfamilies Chalcidoidea and Cynipoidea do not produce any

116 diploid males under inbreeding and must therefore rely on other mechanisms of sex

117 determination [Heimpel and de Boer, 2008]. Unfortunately, current knowledge as to what

118 these are is still very limited. The only alternative mechanism that is reasonably well 119 understood was described in the chalcid wasp Nasonia vitripennis, in which a maternal effect 120 and genomic imprinting are implicated in sex determination [Verhulst et al., 2010].

121 Interestingly, this alternative mechanism revolves around the same principal gene. The 122 maternal copy of the Nasonia vitripennis transformer (Nvtra) gene is transcriptionally 
123 silenced (imprinting), but a maternal input of Nvtra mRNA/protein to the egg is required

124 (maternal effect) to direct female-specific splicing of embryonic Nvtra, which is only

125 expressed in the presence of a paternally derived genome, i.e. in fertilized eggs. Just as in

126 other insects, sex-specific splicing of tra in turn regulates sex-specific splicing of $d s x$ and

127 thus induces male or female development [Beukeboom and van de Zande, 2010; Verhulst et

128 al., 2010].

129

Sex determination affects the evolution of thelytoky

131

132

As introduced above, haplodiploidy predisposes hymenopterans to the evolution of

133 thelytoky, but the sex determination mechanism associated with haplodiploidy may put constraints on such transitions. This is most obvious in the case of gamete duplication and CSD. Independent of whether a single locus or multiple loci are involved in CSD, gamete duplication would lead to complete homozygosity (Fig. 1) and thus to broods consisting

137 entirely of diploid males. Thelytokous parthenogenesis by gamete duplication is therefore

138 considered incompatible with CSD. Gamete duplication is observed when thelytoky is 139 induced by Wolbachia [Stouthamer and Kazmer, 1994; van Wilgenburg et al., 2006], which

140 leads to the prediction that CSD can prevent transitions from arrhenotoky to thelytoky by

141 infection with Wolbachia. This prediction is indeed supported by comparative evidence: even

142 though Wolbachia abounds in the Hymenoptera, it appears that parthenogenesis-inducing

143 strains (PI-Wolbachia) are either restricted to groups in which CSD remains unreported, 144 namely the Chalcidoidea and Cynipoidea [van Wilgenburg et al., 2006], or else to non-CSD 145 species in groups comprising CSD and non-CSD mechanisms of sex determination, such as 146 the Ichneumonoidea [Heimpel and de Boer, 2008; Asplen et al., 2009; Kremer et al., 2009]. It 147 would be premature, however, to conclude that CSD represents an absolute constraint to 
148 microbe-induced thelytoky in general. For one thing, the cytogenetic details of

149 parthenogenesis-induction by Wolbachia have only been worked out for a handful of cases

150 [summarized in van Wilgenburg et al., 2006]. Considering the remarkable diversity of

151 reproductive manipulations exhibited by Wolbachia [Werren et al., 2008], it would not be too

152 surprising if a strain was discovered in the future that overcomes this constraint by an

153 alternative mechanism of parthenogenesis induction. A second reason for caution is that

154 additional microbial endosymbionts like Cardinium or Rickettsia are able to induce thelytoky

155 [Weeks et al., 2003; Hagimori et al., 2006]. In one such case, the Rickettsia inducing

156 thelytoky in the parasitoid wasp Neochrysocharis formosa, the cytological mechanism has

157 been shown to be functionally apomictic and would thus be fully compatible with CSD

158 [Adachi-Hagimori et al., 2008].

159 Forms of thelytoky other than gamete duplication are not or less constrained by CSD.

160 Apomictic or ameiotic parthenogenesis equates to cloning and produces offspring that retain

161 heterozygosity at all loci the mother was heterozygous for (Fig. 1). In species with CSD,

162 independent of the number of loci involved, an apomictic mother will produce daughters

163 only. The evolution of thelytoky by apomixis is thus unconstrained by CSD, yet it may be

164 constrained by sex determination mechanisms that rely on genomic imprinting (see below).

165 Other cytological mechanisms of thelytoky in the Hymenoptera involve the fusion of

166 nuclei after the second meiotic division and are referred to as automixis with terminal (fusion

167 of sister nuclei), central (fusion of non-sister nuclei) or random fusion (Fig. 1). In terms of the

168 loss of heterozygosity in offspring produced by thelytoky, these mechanisms lie between the

169 extreme cases of apomixis (all heterozygosity retained) and gamete duplication (all

170 heterozygosity lost). Therefore, the number of loci involved as well as their location in the

171 genome become important for whether sex determination by CSD can constrain the evolution

172 of thelytoky. In the absence of recombination, the genetic consequences of terminal fusion 
173 would be equivalent to gamete duplication (complete homozygosity), and those of central

174 fusion would be equivalent to apomixis (heterozygosity retained), but under random fusion

175 there would be a $1 / 3$ chance for every heterozygous locus to become homozygous in the

176 offspring. That is because each gene copy will be paired with equal probability to one of the

177 three other gene copies, one of which is identical to the focal copy [Oldroyd et al., 2008;

178 Engelstädter et al., 2011]. Yet recombination does of course occur during the first meiotic

179 division, which alters the genetic consequences of thelytoky by terminal or central fusion.

180 After a crossover event under terminal fusion, heterozygosity is lost between the centromere

181 and the chiasma, but may be retained between the chiasma and the telomere (Fig. 1). With

182 free recombination between the centromere and a heterozygous locus in the mother, the

183 overall probability for this locus to be homozygous in an offspring is $1 / 3$ [Engelstädter et al.,

184 2011]. Thus, a thelytokous lineage with terminal fusion automixis is expected to show a

185 quick erosion of heterozygosity, starting from the centromeres out towards the telomeres of

186 each chromosome. This represents a severe constraint to the evolution of thelytoky in species

187 with CSD. After a crossover under central fusion, heterozygosity is maintained between the

188 centromere and the chiasma, but may be lost distal to the chiasma (Fig. 1). Again, the overall

189 probability for a heterozygous locus to become homozygous in the offspring is $1 / 3$ with free

190 recombination. In contrast to terminal fusion, the loss of heterozygosity over time under

191 central fusion automixis is most rapid at the chromosome ends and progresses towards the

192 centromeres. Importantly, genomic regions that are sheltered from recombination, such as

193 those near the centromere or in chromosomal inversions, can retain heterozygosity

194 indefinitely under central fusion automixis. But note that at least in paracentric inversions,

195 heterozygosity can also be lost under central fusion if crossovers occur between centromere

196 and inversion [Sandrock et al., 2011]. Under random fusion automixis, finally, the average 
probability of a heterozygous locus to become homozygous in the next generation (again 1/3) is unaltered by recombination [Oldroyd et al., 2008].

Based on the above there is a clear ranking in terms of the rate at which thelytokous lineages lose heterozygosity over time among the three modes of automixis: terminal fusion $>$ random fusion $>$ central fusion. More importantly, central fusion automixis most readily permits the long-term maintenance of heterozygosity at least in some regions of the genome. That would make it the form of automictic thelytoky most compatible with CSD. So far there is not enough information available for a comparative analysis, but it is unlikely to be a coincidence that many genetically determined cases of thelyotoky in groups with CSD exhibit central fusion automixis, such as in several species of ants [Pearcy et al., 2006; Kellner and Heinze, 2011; Rabeling et al., 2011; Kronauer et al., 2012; Rabeling and Kronauer, 2013], the cape honeybee [Lattorff et al., 2005; Oldroyd et al., 2008], the ichneumonid parasitoid Venturia canescens [Beukeboom and Pijnacker, 2000] or the braconid parasitoid of aphids, Lysiphlebus fabarum [Belshaw and Quicke, 2003; Sandrock and Vorburger, 2011]. Terminal fusion automixis has been reported for only three species [Comrie, 1938; Smith, 1941; Rössler and Debach, 1973; Mateo Leach et al., 2009], and I am not aware of any documented cases of random fusion automixis in the Hymenoptera. However, it would be premature to exclude the occurrence of this form of automixis in the Hymenoptera, considering that it would be difficult to distinguish it from terminal or central fusion. Central fusion automixis has been observed in other animals such as hybrid fish [Lampert et al., 2007].

In some cases of central fusion automixis in the Hymenoptera, genetic marker data confirmed that parts of the genome do maintain heterozygosity. In L. fabarum, for example, about half of the available microsatellite loci lose heterozygosity quickly in thelytokous lines, but the other half retain heterozygosity [Sandrock et al., 2011]. A thelytokous lineage of the cape honey bee also retains partial heterozygosity at selectively neutral marker loci [Oldroyd 
et al., 2011], although this may at least to some extent be a consequence of linkage to loci under overdominant selection [Goudie et al., 2012].

Above I argued that the evolution of automictic thelytoky is least constrained by CSD if it

225

226

227

228

229

230

231

232

233

234

235

236

237

238

239

240

241

242

243

244

245

246

occurs by central fusion. A related prediction is that the evolution of automictic thelytoky is less constrained by CSD if it involves more than a single CSD locus (ml-CSD). Virtually nothing is known about the location of CSD loci in the few species for which ml-CSD could be inferred [de Boer et al., 2008; de Boer et al., 2012], but under the simplest assumption that these loci are distributed randomly across the genome, ml-CSD would increase the chance that at least one locus is located in a region of the genome that is not or only rarely affected by recombination. That, in turn, would decrease the load imposed by diploid male production on the thelytokous line and increase its chances of persistence.

$$
\text { In addition to the number of CSD loci and their probability to be affected by }
$$

recombination, the fitness of diploid males is also important for the probability of automictic thelytoky to evolve in haplodiploids with CSD. Engelstädter et al. [2011] addressed this problem by modeling the spread of a single, recessive allele determining thelytoky in a sexual haplodiploid population. That reflects the genetic basis of thelytoky in the cape honey bee and in L. fabarum [Lattorff et al., 2005; Sandrock and Vorburger, 2011]. Under the common assumption that diploid males are effectively sterile [Zayed and Packer, 2005], the production of diploid males by thelytokous females did indeed impose a constraint on the spread of a thelytoky-determining allele [Engelstädter et al., 2011]. However, at least in some species, diploid males are viable and fertile [Cowan and Stahlhut, 2004; de Boer et al., 2007; Elias et al., 2009; C. Sandrock \& C. Vorburger, unpubl. data]. If that is the case, diploid male production will promote rather than hamper the evolution of thelytoky, because these males become vehicles for the effective spread of thelytoky-inducing alleles - a mechanism referred 
247 to as contagious parthenogenesis [Simon et al., 2003; Engelstädter et al., 2011]. The

248 possibility that the production of fertile diploid males may aid the spread of thelytoky is a

249 very interesting aspect of genetically determined forms of automixis, but it is unlikely to be

250 realised in more than a few exceptional cases such as L. fabarum [Engelstädter et al., 2011;

251 Sandrock et al., 2011]. In general, the production of diploid males should be considered a

252 burden to the fitness of a thelytokous line. The strength of the constraint imposed by CSD on

253 the evolution of thelytoky is therefore proportional to the rate at which thelytokous lines lose

254 heterozygosity, that is highest under gamete duplication, lowest (absent) under apomixis, and

255 intermediate under the different forms of automixis with recombination. However, we should

256 not forget that the rate of recombination is a trait that can respond to selection [e.g. Kerstes et

257 al., 2012], and by extension the rate of diploid male production under automixis as well. If

258 transitions from arrhenotoky to thelytoky occur repeatedly and different thelytokous lines

259 compete within a population, those with a lower diploid male production will enjoy a higher

260 rate of increase and thus be favoured by selection. This may explain an interesting

261 observation in L. fabarum, a parasitoid species in which the genetic determination of

262 thelytoky by central fusion automixis allows the breeding of new thelytokous lines in the

263 laboratory. Such newly formed lines show a substantial diploid male production of up to one

264 third of the offspring [Engelstädter et al., 2011], whereas thelytokous lines occurring in the

265 field produce very few males, if any. Those males are typically haploid, indicating that they

266 are the result of occasional failures in the fusion of nuclei after the second meiotic division

267 and not of CSD homozygosity, although diploid males are rarely found in the field as well

268 [Sandrock et al., 2011]. Selection for reduced recombination may also explain the case of the

269 little fire ant, Wasmannia auropunctata, in which apomictic production of queens was intially

270 inferred from genotyping data [Fournier et al., 2005], but later found to occur by central

271 fusion automixis with dramatically reduced recombination rates [Rey et al., 2011]. 
273 Could sex determination systems other than CSD also constrain the evolution of thelytoky

274 in the Hymenoptera? The main obstacle to answering this question is the dearth of

275 information on the mechanisms of sex determination in species without CSD. For the one

276 alternative that is well understood, the maternal effect genomic imprinting mechanism

277 described from Nasonia [Verhulst et al., 2010], the answer is certainly yes. At first glance,

278 the requirement of a paternal genome for female development is an absolute constraint for

279 transitions to thelytoky. However, Beukeboom and van de Zande [2010] have argued that the

280 maternal imprint may occur during oogenesis, but not be copied on during the subsequent

281 divisions of the egg, which would allow thelytokous lineages to evolve independence from

282 the contribution of a paternal genome. This conjecture remains to be tested.

283

284 Can thelytoky also influence the evolution of sex determination?

285

While it is clear from the above that the mechanisms of sex determination can constrain

287 the evolution of thelytoky in the Hymenoptera, the opposite question has received less

attention. Can thelytokous reproduction have an impact on the sex determination system? The

model by Engelstädter et al. [2011] mentioned earlier illustrates a route by which this may

290 indeed occur, namely by eroding a ml-CSD system to a sl-CSD system.

291 It has been argued that ml-CSD can evolve from sl-CSD by one or several duplications of

292 the CSD locus and may be favoured by selection because it reduces the inbreeding load 293 resulting from diploid male production [Crozier, 1971; van Wilgenburg et al., 2006; de Boer

294 et al., 2008]. Analogously, I have argued above that ml-CSD may be conducive to the

295 evolution of thelytoky with automixis because it increases the probability that at least one

296 CSD locus occurs in a region of the genome that is sheltered from recombination. However, 
as soon as an automictic line with two or more CSD loci has been formed, an increasing number of loci will become homozygous over time until only one heterozygous CSD locus is left [Engelstädter et al., 2011]. This would normally be the locus with the lowest probability of becoming homozygous, by virtue of being close to a centromere or otherwise sheltered from recombination. Thus, thelytoky with automixis can have a meltdown effect on ml-CSD systems and reduce them to what is effectively sl-CSD. It is worth pointing out that this process can be seen as conceptually related to the sex determination meltdown that may occur in an arrhenotokous population with ml-CSD if a bottleneck is followed by inbreeding [de Boer et al., 2012].

According to Normark [2003] transitions from arrhenotoky to thelytoky are at least in principle reversible. However, this is likely to depend on the number of generations a lineage has reproduced asexually. Over time such lineages can accumulate mutations in genes important for sexual reproduction that will eventually preclude a return to sexual reproduction [e.g. Zchori-Fein et al., 1992]. This is illustrated by the many cases of microbe-

311 induced thelytoky in which antibiotic curing of the symbiont cannot restore functional sexual reproduction [Koivisto and Braig, 2003]. Nevertheless, in the light of the prediction that automictic thelytoky would rapidly melt down ml-CSD to sl-CSD, it is tempting to consider the intriguing possibility that episodes of asexual reproduction, to which hymenopterans are so prone, may influence the evolution of their sex determination systems.

\section{Thelytoky as a window of opportunity to study sex determination} genetic factors can clash with the sex determination of hymenopterans, and how thelytoky -

321 depending on the cytological mechanism involved - may in turn affect the sex determination 
system. It is worth considering whether such dynamics could possibly be used as a window of opportunity to study sex determination. It is difficult and labour-intensive, for example, to experimentally distinguish non-CSD mechanisms of sex determination from ml-CSD, because many generations of inbreeding are required to achieve a level of homozygosity that allows ruling out CSD involving several loci based on the lack of diploid males among inbred offspring [Cook, 1993; de Boer et al., 2008; Ma et al., 2013]. In principle, complete homozygosity could be achieved more quickly if it was possible to introduce a strain of PIWolbachia that induces thelytoky by gamete duplication in the species under investiation. While it is possible to transfer Wolbachia horizontally to new species by microinjection

331 [Grenier et al., 1998], it is less predictable whether it will have the same phenotypic effect in 332 the new genetic background [Fujii et al., 2001; Veneti et al., 2005]. The feasibility of this approach is thus currently uncertain.

Another potential opportunity is offered by the gradual erosion of heterozygosity under automixis. According to the model by Engelstädter et al. [2011], this should lead to the stepwise loss of heterozygosity at all but one CSD locus in species with ml-CSD, and hence in a parallel increase in the proportion of diploid males produced. In species with a simple genetic basis of automictic thelytoky, it may be possible to create new automictic lineages from an outbred population by targeted breeding [Sandrock and Vorburger, 2011]. Similar to

340 multi-generation inbreeding experiments in arrhenotokous species, tracking the rate of

341 diploid male production in such newly formed automictic lines over generations might allow 342 inferences regarding the number of CSD loci a species possesses and the rates at which they 343 acquire homozygosity. Again, this is still a hypothetical scenario and its practical feasibility 344 remains to be tested. 


\section{Conclusions}

Haplodiploidy in the Hymenoptera comes with the ability to initiate development of

unfertilized eggs and entails a low load of recessive deleterious mutations in the genome. As a consequence, hymenopterans are prone to transitions from arrhenotoky to thelytoky, either due to genetic mutation or to infection with parthenogenesis-inducing microbes. However, the evolution of thelytoky can be constrained by the sex determination system. For the ancestral mechanism of sex determination in the Hymenoptera, CSD, the constraint results

354 from the production of low-fitness diploid males when the cytological mechanism of 355 thelytoky involves some form of automixis and hence a loss of heterozygosity at the sex locus (sl-CSD) or loci (ml-CSD). This constraint is absent for apomictic thelytoky. It is therefore surprising that apomixis - generally a common form of thelytoky in insects - is only rarely found in hymenopterans [Mateo Leach et al., 2009]. All other modes of thelytoky are associated with a loss of heterozygosity, and the severity of the constraint imposed by

360 CSD on the evolution of thelytoky is proportional to the rate at which this loss occurs. It

361 follows the order central fusion automixis $<$ random fusion automixis $<$ terminal fusion automixis $<$ gamete duplication. Transitions to thelytoky by gamete duplication are fully constrained by CSD because the loss of heterozygosity is complete, whereas central fusion is

364 the form of automixis most compatible with CSD, because it allows the long-term

365 maintenance of heterozygosity at least in some regions of the genome. Another mechanism of 366 sex determination by a maternal effect and genomic imprinting described from Nasonia 367 [Verhulst et al., 2010] is also likely to constrain the evolution of thelytoky because a paternal genome is required for female development. However, more needs to be known about the mechanism and timing of imprinting before it can be judged whether this constraint cannot be 370 circumvented. Additional mechanisms of hymenopteran sex determination are likely to be 
371 discovered in the future, and it will be interesting to see what constraints, if any, those impose 372 on the evolution fo thelytoky.

373 Interestingly, constraints between sex determination and the evolution of thelytoky may be

374 mutual. Once thelytoky by automixis has evolved in a species with ml-CSD, for example, it

375 may in turn result in the meltdown of ml-CSD to sl-CSD [Engelstädter et al., 2011]. This

376 example shall highlight the more general aspect that the study of thelytoky - although

377 certainly of interest in its own right - may shed light on sex determination, since one of the

378 purposes of this review is to encourage researchers to think about how the frequent

379 occurrence of thelytoky in the Hymenoptera can be exploited to learn more about the

380 mechanisms of sex determination in this fascinating group of insects.

382 Acknowledgements

384 I thank Jan Engelstädter and Christoph Sandrock for instructive discussions on the topic 385 and an anonymous reviewer for very helpful comments on a draft of this paper. I gratefully 386 acknowledge the Swiss National Science Foundation for supporting my research (SNSF 387 Professorship PP00P3_123376). 
Adachi-Hagimori T, Miura K, Stouthamer R: A new cytogenetic mechanism for bacterial endosymbiont-induced parthenogenesis in Hymenoptera. Proc R Soc Lond B 275:26672673 (2008).

Asplen MK, Whitfield JB, de Boer JG, Heimpel GE: Ancestral state reconstruction analysis of hymenopteran sex determination mechanisms. J Evol Biol 22:1762-1769 (2009).

Ball SL: Tychoparthenogenesis and mixed mating in natural populations of the mayfly Stenonema femoratum. Heredity 87:373-380 (2001).

Belshaw R, Quicke DLJ: The cytogenetics of thelytoky in a predominantly asexual parasitoid 399 wasp with covert sex. Genome 46:170-173 (2003).

Beukeboom LW, Pijnacker LP: Automictic parthenogenesis in the parasitoid Venturia canescens (Hymenoptera : Ichneumonidae) revisited. Genome 43:939-944 (2000).

Beukeboom LW, van de Zande L: Genetics of sex determination in the haplodiploid wasp Nasonia vitripennis (Hymenoptera: Chalcidoidea). J Genet 89:333-339 (2010).

Beye M, Hasselmann M, Fondrk MK, Page RE, Omholt SW: The gene csd is the primary 406 signal for sexual development in the honeybee and encodes an SR-type protein. Cell 114:419-429 (2003).

Comrie LC: Biological and cytological observations on tenthredinid parthenogenesis. Nature 142:877-878 (1938).

410 Bethylidae). Heredity 71:130-137 (1993).

411 Cook JM, Butcher RDJ: The transmission and effects of Wolbachia bacteria in parasitoids.

412 Res Popul Ecol 41:15-28 (1999).

413 Cook JM, Crozier RH: Sex determination and population biology in the Hymenoptera.

414 Trends Ecol Evol 10:281-286 (1995).

415 Corley LS, Blankenship JR, Moore AJ: Genetic variation and asexual reproduction in the 416 facultatively parthenogenetic cockroach Nauphoeta cinerea: implications for the evolution 417 of sex. J Evol Biol 14:68-74 (2001).

418 Corley LS, Blankenship JR, Moore AJ, Moore PJ: Developmental constraints on the mode of 419 reproduction in the facultatively parthenogenetic cockroach Nauphoeta cinerea. Evol Dev $420 \quad 1: 90-99$ (1999). 
421 Corley LS, Moore AJ: Fitness of alternative modes of reproduction: developmental

422 constraints and the evolutionary maintenance of sex. Proc R Soc Lond B 266:471-476

423 (1999).

424 Cowan DP, Stahlhut JK: Functionally reproductive diploid and haploid males in an

425 inbreeding hymenopteran with complementary sex determination. Proc Natl Acad Sci U S

426 A 101:10374-10379 (2004).

427 Crozier RH: Heterozygosity and sex determination in haplo-diploidy. Am Nat 105:399-\&

$428 \quad$ (1971).

429 de Boer JG, Kuijper B, Heimpel GE, Beukeboom LW: Sex determination meltdown upon

430 biological control introduction of the parasitoid Cotesia rubecula? Evolutionary

431 Applications 5:444-454 (2012).

432 de Boer JG, Ode PJ, Rendahl AK, Vet LEM, Whitfield JB, Heimpel GE: Experimental

433 support for multiple-locus complementary sex determination in the parasitoid Cotesia

434 vestalis. Genetics 180:1525-1535 (2008).

435 de Boer JG, Ode PJ, Vet LEM, Whitfield JB, Heimpel GE: Diploid males sire triploid

436 daughters and sons in the parasitoid wasp Cotesia vestalis. Heredity 99:288-294 (2007).

437 Duron O, Bouchon D, Boutin S, Bellamy L, Zhou LQ, Engelstädter J, Hurst GD: The

438 diversity of reproductive parasites among arthropods: Wolbachia do not walk alone. BMC

439 Biology 6 (2008).

440 Elias J, Mazzi D, Dorn S: No need to discriminate? Reproductive diploid males in a

441 parasitoid with complementary sex determination. PLOS One 4 (2009).

442 Engelstädter J: Constraints on the evolution of asexual reproduction. BioEssays 30:1138-

4431150 (2008).

444 Engelstädter J, Sandrock C, Vorburger C: Contagious parthenogenesis, automixis, and sex 445 determination meltdown. Evolution 65:501-511 (2011).

446 Fournier D, Estoup A, Orivel RM, Foucaud J, Jourdan H, Le Breton J, Keller L: Clonal

447 reproduction by males and females in the little fire ant. Nature 435:1230-1234 (2005).

448 Fujii Y, Kageyama D, Hoshizaki S, Ishikawa H, Sasaki T: Transfection of Wolbachia in

449 Lepidoptera: the feminizer of the adzuki bean borer Ostrinia scapulalis causes male killing

450 in the Mediterranean flour moth Ephestia kuehniella. Proc R Soc Lond B 268:855-859

451 (2001).

452 Gempe T, Beye M: Function and evolution of sex determination mechanisms, genes and

453 pathways in insects. BioEssays 33:52-60 (2011). 
Gempe T, Hasselmann M, Schiott M, Hause G, Otte M, Beye M: Sex determination in

455

456

457

458

459

460

461

462

463

464

465

466

467

468

469

470

471

472

473

474

475

476

477

478

479

480

481

482

483

484

485

486

487

honeybees: Two separate mechanisms induce and maintain the female pathway. Plos Biology 7 (2009).

Gerritsen J: Sex and parthenogenesis in sparse populations. Am Nat 115:718-742 (1980).

Goudie F, Allsopp MH, Beekman M, Oxley PR, Lim J, Oldroyd BP: Maintenance and loss of heterozygosity in a thelytokous lineage of honey bees (Apis mellifera capensis). Evolution 66:1897-1906 (2012).

Grenier S, Pintureau B, Heddi A, Lassabliere F, Jager C, Louis C, Khatchadourian C: Successful horizontal transfer of Wolbachia symbionts between Trichogramma wasps. Proc R Soc Lond B 265:1441-1445 (1998).

Grimaldi D, Engel MS: Evolution of the Insects, (Cambridge University Press, Cambridge, UK 2005).

Hagimori T, Abe Y, Date S, Miura K: The first finding of a Rickettsia bacterium associated with parthenogenesis induction among insects. Curr Microbiol 52:97-101 (2006).

Hamilton WD: Genetical evolution of social behaviour II. J Theor Biol 7:17-52 (1964).

Hasselmann M, Gempe T, Schiott M, Nunes-Silva CG, Otte M, Beye M: Evidence for the evolutionary nascence of a novel sex determination pathway in honeybees. Nature 454:519-U517 (2008).

Heimpel GE, de Boer JG: Sex determination in the Hymenoptera. Annu Rev Entomol 53:209-230 (2008).

Holloway AK, Heimpel GE, Strand MR, Antolin MF: Survival of diploid males in Bracon sp. near hebetor (Hymenoptera : Braconidae). Ann Entomol Soc Am 92:110-116 (1999).

Kellner K, Heinze J: Mechanism of facultative parthenogenesis in the ant Platythyrea punctata. Evol Ecol 25:77-89 (2011).

Kerstes NAG, Berenos C, Schmid-Hempel P, Wegner KM: Antagonistic experimental coevolution with a parasite increases host recombination frequency. BMC Evol Biol 12 (2012).

Koivisto RKK, Braig HR: Microorganisms and parthenogenesis. Biol J Linn Soc 79:43-58 (2003).

Kraaijeveld K: Male genes with nowhere to hide; sexual conflict in haplodiploids. Anim Biol 59:403-415 (2009).

Kremer N, Charif D, Henri H, Bataille M, Prevost G, Kraaijeveld K, Vavre F: A new case of Wolbachia dependence in the genus Asobara: evidence for parthenogenesis induction in Asobara japonica. Heredity 103:248-256 (2009). 
488

489

490

491

492

493

494

495

496

497

498

499

500

501

502

503

504

505

506

507

508

509

510

511

512

513

514

515

516

517

518

519

520 521 JHC, Mariano CDF, Estoup A: Meiotic recombination dramatically decreased in

Kronauer DJC, Pierce NE, Keller L: Asexual reproduction in introduced and native populations of the ant Cerapachys biroi. Mol Ecol 21:5221-5235 (2012).

Lampert KP, Lamatsch DK, Fischer P, Epplen JT, Nanda I, Schmid M, Schartl M: Automictic reproduction in interspecific hybrids of Poeciliid fish. Curr Biol 17:1948-1953 (2007).

Lattorff HMG, Moritz RFA, Fuchs S: A single locus determines thelytokous parthenogenesis of laying honeybee workers (Apis mellifera capensis). Heredity 94:533-537 (2005).

Ma WJ, Kuijper B, de Boer JG, van de Zande L, Beukeboom LW, Wertheim B, Pannebakker BA: Absence of complementary sex determination in the parasitoid wasp genus Asobara (Hymenoptera: Braconidae). Plos One 8 (2013).

Mateo Leach I, Pannebakker BA, Schneider MV, Driessen G, Van de Zande L, Beukeboom LW: Thelytoky in Hymenoptera with Venturia canescens and Leptopilina clavipes as case studies, in Schön I. Martens K. Van Dijk P (eds): Lost Sex - The Evolutionary Biology of Parthenogenesis, pp 347-375 (Springer, Dordrecht Heidelberg London New York 2009).

Normark BB: The evolution of alternative genetic systems in insects. Annu Rev Entomol 48:397-423 (2003).

Oldroyd BP, Allsopp MH, Gloag RS, Lim J, Jordan LA, Beekman M: Thelytokous parthenogenesis in unmated queen honeybees (Apis mellifera capensis): Central fusion and high recombination rates. Genetics 180:359-366 (2008).

Oldroyd BP, Allsopp MH, Lim J, Beekman M: A thelytokous lineage of socially parasitic honey bees has retained heterozygosity despite at least 10 years of inbreeding. Evolution 65:860-868 (2011).

Pardo MC, Lopezleon MD, Cabrero J, Camacho JPM: Cytological and developmental analysis of tychoparthenogenesis in Locusta migratoria. Heredity 75:485-494 (1995).

Pearcy M, Hardy O, Aron S: Thelytokous parthenogenesis and its consequences on inbreeding in an ant. Heredity 96:377-382 (2006).

Rabeling C, Gonzales O, Schultz TR, Bacci M, Garcia MVB, Verhaagh M, Ishak HD,

15 Mueller UG: Cryptic sexual populations account for genetic diversity and ecological 6 success in a widely distributed, asexual fungus-growing ant. Proc Natl Acad Sci U S A 108:12366-12371 (2011).

18 Rabeling C, Kronauer DJC: Thelytokous parthenogenesis in eusocial Hymenoptera. Annu 19 Rev Entomol 58:273-292 (2013).

20 Rey O, Loiseau A, Facon B, Foucaud J, Orivel J, Cornuet JM, Robert S, Dobigny G, Delabie 
thelytokous queens of the little fire ant and their sexually produced workers. Mol Biol Evol 28:2591-2601 (2011).

524 Rössler Y, Debach P: Genetic variability in a thelytokous form of Aphytis mytilaspidis (Le 525 Baron) (Hymenoptera: Aphelinidae). Hilgardia 42:149-175 (1973).

526 Sandrock C, Schirrmeister BE, Vorburger C: Evolution of reproductive mode variation and 527 host associations in a sexual-asexual complex of aphid parasitoids. BMC Evol Biol 11:348 528 (2011).

529 Sandrock C, Vorburger C: Single-locus recessive inheritance of asexual reproduction in a parasitoid wasp. Curr Biol 21:433-437 (2011).

531 Schwander T, Vuilleumier S, Dubman J, Crespi BJ: Positive feedback in the transition from 532 sexual reproduction to parthenogenesis. Proc R Soc Lond B 277:1435-1442 (2010).

533 Sekine K, Tojo K: Potential for parthenogenesis of virgin females in a bisexual population of 534 the geographically parthenogenetic mayfly Ephoron shigae (Insecta: Ephemeroptera, 535 Polymitarcyidae). Biol J Linn Soc 99:326-334 (2010).

536 Shuker DM, West SA: Information constraints and the precision of adaptation: Sex ratio manipulation in wasps. Proc Natl Acad Sci U S A 101:10363-10367 (2004).

538 Simon JC, Delmotte F, Rispe C, Crease T: Phylogenetic relationships between parthenogens and their sexual relatives: the possible routes to parthenogenesis in animals. Biol J Linn

541 Smith SG: A new form of the spruce sawfly identified by means of its cytology and 542 parthenogenesis. Sci Agric 21:245-305 (1941).

543 Snell GD: The determination of sex in Habrobracon. Proc Natl Acad Sci U S A 21:446-453 (1935).

545 Stouthamer R, Kazmer DJ: Cytogenetics of microbe-associated parthenogenesis and its 546 consequences for gene flow in Trichogramma wasps Heredity 73:317-327 (1994).

van Wilgenburg E, Driessen G, Beukeboom LW: Single locus complementary sex determination in Hymenoptera: an "unintelligent" design? Front Zool 3:1 (2006).

Veneti Z, Bentley JK, Koana T, Braig HR, Hurst GDD: A functional dosage compensation complex required for male killing in Drosophila. Science 307:1461-1463 (2005).

553 Weeks AR, Velten R, Stouthamer R: Incidence of a new sex-ratio-distorting endosymbiotic 554 bacterium among arthropods. Proc R Soc Lond B 270:1857-1865 (2003). 
555 Werren JH: The evolution of inbreeding in haplodiploid organisms, in Thornhill NW (ed)

556 The Natural History of Inbreeding and Outbreeding: Theoretical and Empirical

557 Perspectives, pp 42-94 (University of Chicago Press, Chicago 1993).

558 Werren JH, Baldo L, Clark ME: Wolbachia: master manipulators of invertebrate biology.

559 Nature Reviews Microbiology 6:741-751 (2008).

560 Whiting PW: Selective fertilization and sex-determination in Hymenoptera. Science 78:537$561538(1933)$.

562 Whiting PW: Sex determination and reproductive economy in Habrobracon. Genetics $56324: 110-111(1939)$.

564 Whiting PW: Multiple alleles in complementary sex determination of Habrobracon. Genetics 565 28:365-382(1943).

566 Zayed A, Packer L: Complementary sex determination substantially increases extinction

567 proneness of haplodiploid populations. Proc Natl Acad Sci U S A 102:10742-10746 568 (2005).

569 Zchori-Fein E, Roush RT, Hunter MS: Male production induced by antibiotic treatment in 570 Encarsia formosa (Hymenoptera: Aphelinidae), an asexual species. Experientia 48:102571105 (1992).

572 


\section{$573 \quad$ Figure captions}

574

575 Fig. 1. Modes of thelytoky in the Hymenoptera. Under apomixis, all cell divisions are mitotic

576 and no recombination takes place. Under the other modes, crossing-over may occur during

577 the first meiotic division. The figure illustrates the fate of a heterozygous locus in the mother

578 that is $(\mathrm{B} / \mathrm{b})$ or is not $(\mathrm{A} / \mathrm{a})$ affected by recombination. One fusion product is illustrated as an

579 example, but four different products are possible under central fusion or gamete duplication,

580 and two under terminal fusion, depending on which nuclei fuse or duplicate, respectively. Not

581 shown in the figure is random fusion automixis, under which any two of the four nuclei could

582 fuse to form a diploid zygoid. This form of automixis might also occur in the Hymenoptera

583 but has so far not been documented unambiguously. 


\section{$585 \quad$ Figure 1}

586
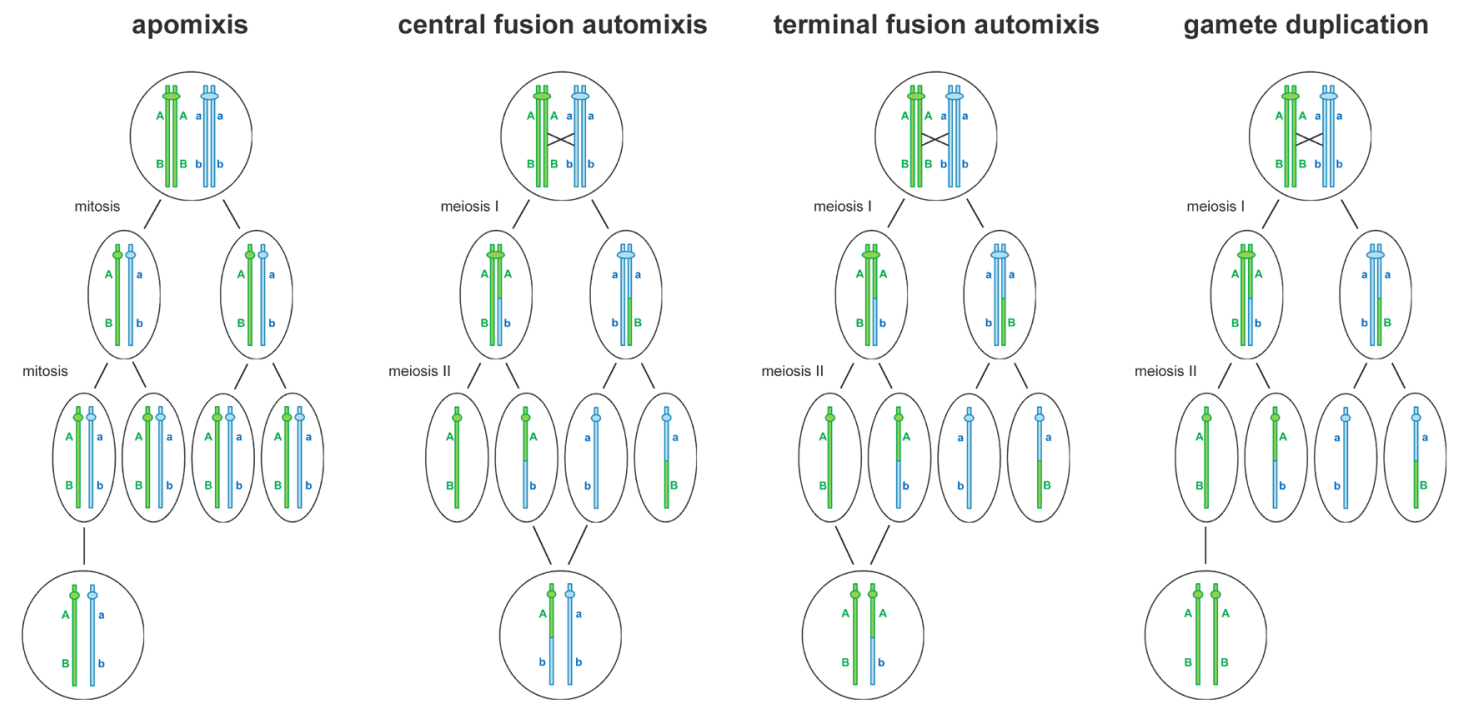